\title{
Originales
}

\section{Carga mental en la mujer trabajadora: desigualdad de género y prevalencia}

\section{Mental load in working women: gender inequality and prevalence}

\author{
M. ${ }^{a}$ Carmen Fernández Felipe ${ }^{1}$, M. ${ }^{a}$ Luisa de la Cruz Cantos $^{2}$, María Gayoso Doldan ${ }^{3}$, Shirley Rodríguez Tupayachi ${ }^{4}$ \\ 1. Servicio de Prevención. Hospital Universitario de la Princesa. Madrid. España. \\ 2. Servicio de Prevención. Hospital Universitario de Getafe. Madrid. España. \\ 3. Mutua Universal. Madrid. España. \\ 4. Servicio de Prevención. Hospital Universitario Fundación Alcorcón. Madrid. España
}

Recibido: 19-12-14

Aceptado: 23-12-14

\section{Correspondencia}

M. ${ }^{a}$ Carmen Fernández Felipe

medca@telefonica.net

Este artículo se realiza dentro del Programa de Investigación MIR de la Escuela Nacional de Medicina del Trabajo, del Instituto de Salud Carlos III; Madrid, España

Resumen

Introducción: La igualdad de género hay sido una preocupación introducida en los últimos años por instituciones tanto internacionales como nacionales. La cuestión de igualdad afecta también al ámbito laboral tanto en lo que se refiere a la distribución del trabajo como a la exposición a riesgos psicosociales, principalmente en economías basadas en la información y conocimiento.

Los objetivos de este estudio se dirigen a identificar, entre la población trabajadora española, situaciones de desigualdad entre géneros en lo que se refiere a los diferentes componentes de la carga mental y estimar su prevalencia.

Material y métodos: Se realizó un estudio observacional de tipo transversal a partir de los datos recogidos de la "VII Encuesta Nacional de Condiciones del Trabajo" con una muestra de 8.892 trabajadores. Los datos se analizaron de forma bivariante utilizándose el test de Chi-Cuadrado para las variables cualitativas y el t-test para muestras independientes en el caso de variables cuantitativas. La significación se consideró con p $<0,05$.

Con el objeto de analizar la influencia de distintos factores potencialmente relacionados con componentes de la carga mental y su interacción, se realizó un análisis de regresión logística binaria con el método de inclusión retroceder por paso (Wald).

Resultados: Los hombres manifiestan sufrir una menor carga mental que la mujer en relación con el ritmo de trabajo, OR = 0,89 (0,8-0.9), la prevalencia de un "Alto ritmo de trabajo» fue de $(0,65 \%$ ) en mujeres con respecto a un $0,63 \%$ en hombres. Los hombres refirieron una menor carga mental por "exceso de trabajo" con respecto a las mujeres $\mathrm{OR}=0,9(0,8-1,0)$. Las mujeres con mayor frecuencia que el hombre se encuentran fatigadas por una sobrecarga de trabajo $(0,37 \%) ; 0,35 \%$ ) respectivamente.

Conclusiones: Estos resultados no corroboran nuestra hipótesis inicial de que la mujer está expuesta a una mayor carga mental que el hombre. Analizando diferentes componentes de la carga mental, la mujer está expuesta a un mayor ritmo de trabajo y a una mayor fatiga como consecuencia del a cantidad de trabajo.

Med Segur Trab (Internet) 2015; 61 (238) 18-33

Palabras clave: Desigualdad, género, trabajo, condiciones de trabajo, mujer, carga mental. 


\section{Abstract}

Introduction: Gender equality is a concern introduced in the last years by both, international and national institutions. Equality is also an issue into the world of work, involving job division and occupational exposure to psychosocial risks, mainly in societies which economy is based in information and knowledge.

The aims are to identify, among the Spanish working population, gender inequality situations regarding the different components of mental load and to estimate its prevalence.

Methods: An observational cross-sectional study was conducted using data from the Spanish «VII National Survey on Working Conditions» with a sample of 8.892 workers. The data were analyzed in a bi-variant way, chi-square test was used for qualitative variables and t-test for independent samples for quantitative variables. The significance was considered at $\mathrm{p}<0.05$.

In order to analyze the influence of any factors related with mental workload, its components and their interaction, a binary logistic regression was performed using step backward method (Wald).

Results: Men have a lower mental load than women regarding work pace, OR =0,89 (0,8-0.9), prevalence of a "high work pace» was ( 0,65\%) in women versus (0,63\%o) in men. Men also refer lower "work load" than women OR $=0,9(0,8-1,0)$, figures show, for women, an higher prevalence of fatigue due to work load exposure than men $(0,37 \%$ ) versus $0,35 \%$ )

Conclusion: These results did not corroborate our hypothesis, that women are exposed to a higher overall mental load that men. Analyzing several components of mental load women are more exposed to an «high work pace» and an «high work load and fatigue».

Med Segur Trab (Internet) 2015; 61 (238) 18-33

Keywords: Inequality, Gender, working conditions, mental load. 


\section{INTRODUCCIÓN}

El empleo, conforme al Convenio núm. 111 sobre discriminación, empleo y ocupación de 1958 de la OIT, que establece la eliminación de toda forma de discriminación, es un derecho fundamental de todos, que en el caso de la mujer todavía permanece sumergido en la desigualdad, a pesar de existir una amplia normativa en el ámbito nacional e internacional, que vincula las acciones de las entidades públicas y privadas con la igualdad de género garantizando el pleno acceso al mercado laboral, derecho al empleo remunerado, educación, formación vocacional, promoción en el trabajo y seguridad del empleo.

Desde esta fecha, la cuestión de género y la no discriminación ha sido objeto de atención por parte de organismos internacionales, como la IV Conferencia de la ONU sobre la Mujer, celebrada Pekín en $1995^{1}$.

En el marco jurídico europeo, el Tratado de Ámsterdam en 1972, ratificado por España en 1999, promueve la inclusión de la cuestión de igualdad de oportunidades en distintas políticas. De acuerdo a este tratado, todas las acciones políticas, programas, proyectos o toma de decisiones desarrolladas desde los Gobiernos deben promover la igualdad de oportunidades entre mujeres y hombres ${ }^{3}$.

Se introduce así, el concepto de género como categoría de análisis, que permite diferenciar y separar lo biológico, atribuido al sexo, de lo cultural que determina las funciones, actitudes, valores y relaciones que conciernen al hombre, a la mujer y a la igualdad de género.

El Consejo Europeo insta a los Estados Miembros a implementar la perspectiva de género, e inicia un plan de trabajo donde se adoptan una serie de medidas para acelerar el progreso de una igualdad real entre ambos sexos. Propone acciones en varias áreas prioritarias: igualdad, independencia económica, conciliación de la vida privada y la actividad profesional; igual representación en la toma de decisiones; erradicación de todas las formas de violencia de género y de trato de seres humanos; la eliminación de los estereotipos sexistas; y la promoción de la igualdad de género fuera de la Unión Europea $^{4}$.

Posteriormente en España la Ley Orgánica 3/2007, de 22 de marzo, tiene por objeto hacer cumplir el derecho de igualdad mediante la eliminación de la discriminación de la mujer, estableciendo que las mujeres y los hombres son iguales en dignidad humana, en derechos y deberes para, alcanzar una sociedad más justa ${ }^{5}$.

Justicia e igualdad para hombres y mujeres incluidos los jóvenes, promover el trabajo decente y productivo en condiciones de libertad, equidad, seguridad y dignidad humana con aspiraciones en relación a oportunidades laborales; salariales; derecho a voz; reconocimiento; estabilidad familiar y desarrollo personal son parte del Programa de Trabajo Decente de la OIT. Todos los trabajadores tienen derecho a un trabajo decente, no sólo los que trabajan en la economía formal e informal, sino también los trabajadores independientes, así como aquellos, en su mayoría mujeres que trabajan en la economía del cuidado de personas y hogares privados ${ }^{6}$.

Por lo tanto, existe una normativa internacional, europea y nacional que obliga a la incorporación de esta perspectiva de género en las políticas públicas. En este sentido hay autores que plantean que la promoción de la igualdad de género y el dialogo social son mutuamente beneficiosos ${ }^{6}$. La importancia de considerar las diferencias entre hombres y mujeres para reconocer los factores que pueden influir en los peligros a los que se enfrentan en el puesto de trabajo, se traduce en adoptar estrategias de prevención adecuadas "desde una perspectiva de género" para las cuestiones de la salud y la seguridad en el trabajo ${ }^{7}$.

Valentina Forastieri, recomienda que las políticas de promoción de la salud construidas dentro de Política Nacional de Seguridad y Salud Ocupacional para las 
mujeres que trabajan, deben tener en cuenta tres funciones: como amas de casa, como madres y como trabajadoras $^{8}$.

En el artículo de Wu SY, sobre una comparación entre los efectos del estrés laboral y la calidad de vida entre las enfermeras y mujeres médicos, se identificaron factores desencadenantes del estrés, que se deben tener en cuenta en el mundo laboral porque juegan un papel importante en el agotamiento durante el trabajo y en la calidad de vida de las enfermeras y las mujeres médicos?.

En mujeres que ocupaban puestos profesionales como especialistas, con algún cargo directivo o como ejecutivos con responsabilidades de gestión, fueron identificados algunos factores de estrés tantos externos como internos. Sobre la base de estos hallazgos, se concluye la importancia de prestar atención a la identificación de indicadores tempranos de agotamiento y se deben tomar medidas preventivas en el ámbito laboral y en la vida en su conjunto para promover la capacidad de trabajo ${ }^{10}$.

En el empleo, el 10\% de los ingresos del mundo los recibe la mujer, pero realizan dos tercios del trabajo mundial no remunerado ${ }^{11}$.

Miguel Toharia ha hecho hincapié en las diferencias entre la evolución del empleo, el paro de los hombres y de las mujeres. Aunque éstas han experimentado un crecimiento del empleo más rápido y persistente, representando más de la mitad del empleo total creado, su posición sigue siendo de mayor nivel de paro, casi el doble que el de los hombres. El esfuerzo de creación de empleo debe ser, pues, especialmente intenso en lo que se refiere a las mujeres ${ }^{12}$.

Samuelsson Å y cols., en el estudio realizado sobre la relación entre la salud de mujeres y hombres suecos y los tipos de empleo o trabajos, obtuvieron como resultado que la pobre percepción de salud y el agotamiento emocional, estaban significativamente asociados a las características del trabajo que realizaban ${ }^{13}$.

M. ${ }^{a}$ del Mar García Calvente, informa que la investigación en salud pública, arrastra tradicionalmente la ausencia en el tratamiento de la cuestión de género en sus diseños, derivando en la generalización de los resultados a ambos sexos. Con una incorporación insuficiente de variables y categorías de análisis relacionadas con el género ${ }^{14}$.

En el estudio de Mota N P., sobre los trastornos mentales y estrés en el personal militar femenino, las comparaciones entre los sexos en una profesión dominada por los hombres, concluyó que a pesar que la proporción de mujeres en los ejércitos está creciendo, los estudios de salud mental en el ejército se han realizado sobre muestras de mayoría masculina ${ }^{15}$.

Campos- Serna, J., nos transmite que en España existen importantes desigualdades de género, tanto en las condiciones de empleo, condiciones del ambiente de trabajo, como problemas de salud relacionados con el trabajo y conciliación de la vida laboral y familiar. Todo ello influido por la clase social y el sector de actividad, aspectos necesarios a tener en consideración en el desarrollo de las políticas públicas ${ }^{20}$.

En base a estos antecedentes, el presente estudio se dirige a contrastar la hipótesis de desigualdad, en contra de la mujer, en relación a la carga mental en el trabajo. Teniendo como objetivo principal identificar aquellos componentes de la carga mental como:» ritmo de trabajo", "nivel de atención", "trabajar muy rápido y en plazos muy estrictos", "carga de trabajo", "sentirse agobiado", en cuya distribución puede existir una desigualdad entre géneros. Son objetivos secundarios estimar la prevalencia en población trabajadora femenina en estos aspectos. El estudio se basa en el análisis, bajo el enfoque de género y trabajo, de la información suministrada por la VII Encuesta Nacional de Condiciones de Trabajo, del INSHT ${ }^{17}$. 


\section{MATERIAL Y MÉTODOS}

Al objeto de proceder a la verificación de la hipótesis planteada y a la consecución de los objetivos formulados, se ha diseñado un estudio observacional transversal de fuente secundaria, a partir de los datos de la «VII Encuesta Nacional de Condiciones del Trabajo", realizada en el año 2011 por el Instituto Nacional de Seguridad e Higiene en el Trabajo.

El trabajo de campo de la VII E. N. C. T. se realizó entre Octubre 2011 y Febrero de 2012. La encuesta cubre el ámbito geográfico de todo el territorio nacional a excepción de Ceuta y Melilla. Se ha realizado sobre una muestra representativa a nivel nacional con 8.892 trabajadores activos a partir de 16 años, de todas las actividades económicas, mediante entrevista personal en el domicilio del trabajador.

La encuesta consta de 14 apartados: situación laboral y tipo de contrato, datos del centro de trabajo, tipo de trabajo, agentes físicos, contaminantes químicos biológicos, condiciones de seguridad, diseño del puesto, carga de trabajo y factores psicosociales, organización de la prevención, horario de trabajo, actividades preventivas, información/ formación, conductas violentas en el trabajo, daños a la salud y datos personales en un total 62 preguntas.

Para este estudio, se incluyeron aquellas variables que indicaran el nivel de carga mental, así como aquellas descriptivas de la población (tabla 1).

Tabla 1. Variables de la VII ENCT incluidas en el estudio

\begin{tabular}{lll}
\hline & & TARIABLE \\
\hline Edad & Cuantitativa & Años \\
\hline Sexo & Dicotómica & Hombre, mujer \\
\hline Nacionalidad & Politómica & País de nacimiento \\
\hline Nivel atención & Categórica ordinal & Escala tipo Likert de 5 categorías \\
\hline Trabajar en plazos muy estrictos y muy cortos & Categórica ordinal & Escala tipo Likert de 5 categorías \\
\hline Trabajar muy rápido & Categórica ordinal & Escala tipo Likert de 5 categorías \\
\hline Tener mucho trabajo y sentirse agobiado & Categórica ordinal & Escala tipo Likert de 5 categorías \\
\hline Nivel de Estudios & Categórica ordinal & 9 categorías \\
\hline Tipo de contrato & Politómica & 8 categorías \\
\hline Tipo de jornada & Politómica & 3 categorías \\
\hline Horario habitual & Politómica & 7 categorías \\
\hline Tipo de puesto de trabajo & Politómica & 5 categorías \\
\hline Actividades principal del lugar de trabajo & Politómica & 11 categorías \\
\hline Obtener ayuda de sus compañeros & Categórica ordinal & Escala tipo Likert de 5 categorías \\
\hline Obtener ayuda de sus superiores /jefes & Categórica ordinal & Escala tipo Likert de 5 categorías \\
\hline Tener la oportunidad de hacer aquello que sabe & Categórica ordinal & Escala tipo Likert de 5 categorías \\
hacer mejor & & \\
\hline Poner en prácticas sus propias ideas & Categórica ordinal & Escala tipo Likert de 5 categorías \\
\hline Compaginar el trabajo con su vida social y familiar & Categórica ordinal & 4 categorías \\
\hline
\end{tabular}

Los indicadores de carga mental utilizados fueron los siguientes: "Nivel de Atención alto o muy alto", "Trabajar muy rápido", "Trabajar en plazos estrictos o cortos" y "Tener mucho trabajo y sentirse agobiado".

Los siguientes factores se utilizaron como indicadores de factores protectores:»Obtener ayuda de sus compañeros si la pide», "Ayuda de sus superiores», "Tener la oportunidad de hacer aquello que sabe hacer mejor» $\mathrm{y}$ «Poner en prácticas sus propias ideas». 
Del estudio se excluyeron aquellos registros que no tenían información completa, en las preguntas o indicadores objeto de análisis.

Se realizó un análisis descriptivo, calculando las frecuencias absolutas y relativas para las variables cualitativas, la media y parámetros de dispersión para la edad.

En el análisis bivariante, para la determinación de la desigualdad entre géneros, se utilizó el test de Chi-Cuadrado para las variables cualitativas y el t-test para muestras independientes para variables cuantitativas. La significación se consideró con una p<0,05.

Los factores de carga mental y factores protectores se dicotomizaron, unificando las categorías "siempre y a menudo" por un lado y "nunca y raramente por otro". Esta dicotomización, fuerza el análisis de forma más desfavorable a nuestra hipótesis de desigualdad en el trabajo según género.

Con el objeto de analizar la influencia de distintos factores potencialmente relacionados con componentes de la carga mental y su interacción, se realizó un análisis de regresión logística binaria con el método de inclusión retroceder por paso (Wald), en la que las variables dependientes fueron: "Mantener un nivel de atención alto o muy alto", "Trabajar muy rápido", "Trabajar con plazos muy estrictos y muy cortos» y "Tiene mucho trabajo y se siente agobiado".

Los valores de estas variables originales (tipo likert de 5 categorías) se dicotomizaron, considerando como exposición los valores de las variables menores a 3 (categorías «a menudo" y "siempre o casi siempre») y como no exposición valores superiores a 3 (categorías "raramente» y "casi nunca o nunca»), el valor medio de la escala se consideró como valor perdido.

La tabla 2 refleja las variables independientes incluidas en los modelos. Aquellas variables independientes politómicas se incluyeron como variables dummy.

La significación de los modelos se contrastó mediante la prueba de Hosmer y Lemeshow.

Tabla 2. Variables independientes incluidas en el estudio

\begin{tabular}{lll}
\hline \multicolumn{1}{c}{ VARIABLE INDEPENDIENTE } & CATEGORÍA DE REFERENCIA & \multicolumn{1}{c}{ TIPO DE VARIABLE } \\
\hline Plantilla del centro de trabajo & 1 a 10 & Dummy \\
\hline Nivel de estudio & Sin estudios & Dummy \\
\hline Trabajar en sector servicios & No servicios & Dicotómica \\
\hline Nacionalidad & Español & Dicotómica \\
\hline Existencia delegado de prevención & Con delegado & Dicotómica \\
\hline Tipo de contrato & Fijo & Dicotómica \\
\hline Tipo de jornada & Tiempo completo & Dicotómica \\
\hline Trabajar en sector construcción & No construcción & Dicotómica \\
\hline Trabajar en sector industria & No industria & Dicotómica \\
\hline Trabajar en sector agrícola & No agrícola & Dicotómica \\
\hline Puede obtener ayuda de jefes o superiores & Con ayuda & Dicotómica \\
\hline Género & Varón & Dicotómica \\
\hline
\end{tabular}

La estimación de la asociación se realizó mediante el cálculo de la Odds Ratio (OR), calculándose sus Intervalos de Confianza al 95 \%. Para el análisis de los datos se utilizó el paquete estadístico SPSS 22.0.0 


\section{RESULTADOS}

La muestra estudiada se caracteriza por una distribución similar entre géneros (gráfico 1), con un discreto predominio del varón. Estas diferencias son menores en intervalos de edad inferior a 34 años.

Gráfico 1. Distribución de la muestra según género
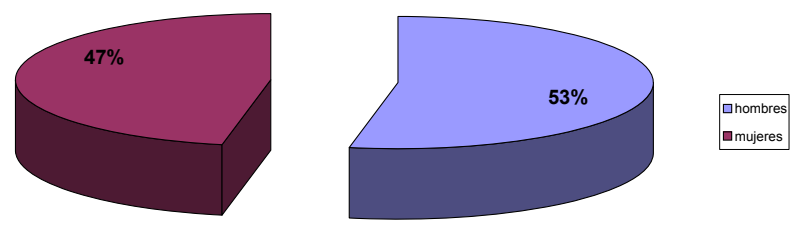

La distribución de la muestra según edad y género (gráfico 2), pone de manifiesto que al comienzo de la vida laboral el porcentaje de hombres y mujeres es muy similar, observándose que a partir de los 25 años hay un descenso mantenido en el peso específico de la población femenina en activo.

Gráfico 2. Distribución de la población según actividad económica y género

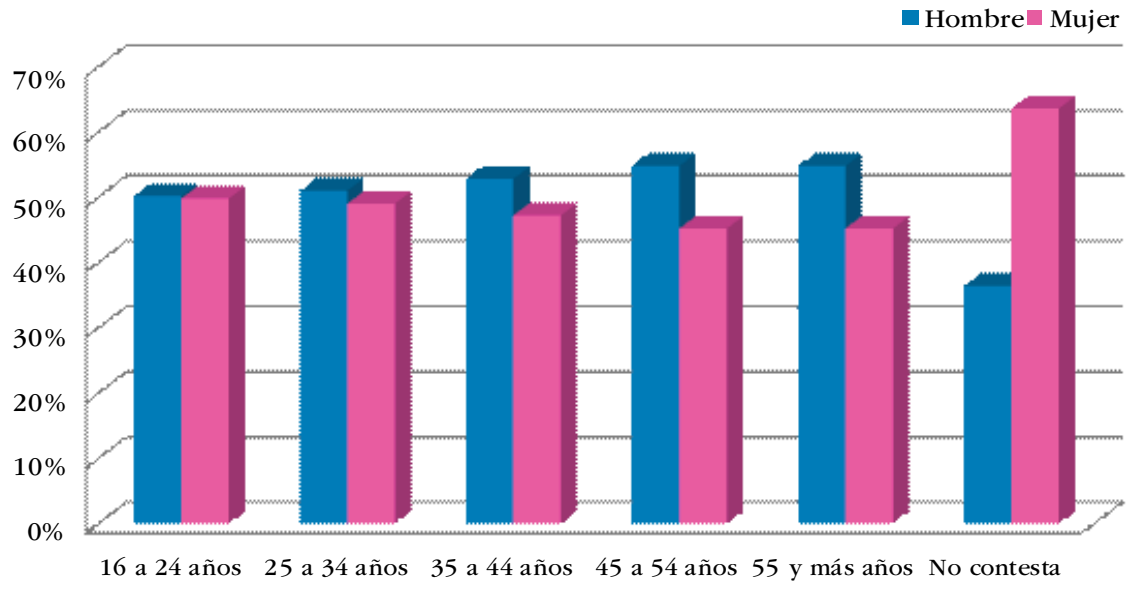

Encontramos que en la muestra es muy superior el número de trabajadores con nacionalidad española con respecto a los extranjeros, hecho que se produce tanto en hombres como en mujeres (tabla 3).

Tabla 3. Distribución de la población según nacionalidad.

\begin{tabular}{lccrc}
\hline & \multicolumn{2}{c}{ HOMBRE } & \multicolumn{2}{c}{ MUJER } \\
\cline { 2 - 5 } & $\mathbf{n}$ & $\%$ & \multicolumn{1}{c}{$\mathbf{n}$} & $\%$ \\
\hline Español & 4.294 & 53,3 & 3.764 & 46,7 \\
\hline Extranjero & 425 & 51,6 & 399 & 48,4 \\
\hline
\end{tabular}


En cuanto al tipo de puesto de trabajo (tabla 4), observamos que la categoría de"empleado" entre hombres y mujeres es muy similar, pero a medida que ascendemos en la escala profesional, observamos un claro predominio en los hombres, esto también ocurre en trabajadores autónomos.

Tabla 4. Distribución de la población según género y tipo de puesto de trabajo

\begin{tabular}{lrrrr}
\hline \multirow{2}{*}{ PUESTO DE TRABAJO } & \multicolumn{2}{c}{ HOMBRE } & \multicolumn{2}{c}{ MUJER } \\
\cline { 2 - 5 } & $\mathbf{n}$ & $\%$ & $\mathbf{n}$ & $\%$ \\
\hline Empleado (con jefes y sin subordinados). & 3.006 & 47,9 & 3.265 & 52,1 \\
\hline Encargado, jefe de taller o de oficina, capataz o similar. & 367 & 70,0 & 157 & 30,0 \\
\hline $\begin{array}{l}\text { Mando intermedio, director de pequeña empresa, } \\
\text { departamento o sucursal. }\end{array}$ & 373 & 63,9 & 211 & 36,1 \\
\hline Director de empresa grande o media. & 47 & 77,0 & 14 & 23,0 \\
\hline Autónomo sin jefe y subordinado. & 656 & 63,6 & 375 & 36,4 \\
\hline
\end{tabular}

En relación con la actividad económica (tabla 5), se evidencia el predominio de la mujer en los sectores de: Administraciones Públicas, Educación, Hostelería y Actividades Culturales. Con predominio en el hombre en los sectores de la Construcción, Transporte, Almacenamiento y Metal.

Tabla 5. Distribución de la población según actividad económica y género

\begin{tabular}{lccrc}
\hline \multirow{2}{*}{\multicolumn{1}{c}{ ACTIVIDAD ECONÓMICA }} & \multicolumn{2}{c}{ HOMBRE } & \multicolumn{2}{c}{ MUJER } \\
\cline { 2 - 5 } & $\mathbf{n}$ & $\%$ & $\mathbf{n}$ & $\%$ \\
\hline Agricultura, ganadería, silvicultura y pesca & 238 & 67,8 & 113 & 32,2 \\
\hline Industria química, saneamiento y extractiva & 193 & 79,4 & 50 & 20,6 \\
\hline Metal & 374 & 84,0 & 71 & 16,0 \\
\hline Industria manufacturera & 332 & 62,2 & 202 & 37,8 \\
\hline Construcción. & 635 & 91,9 & 56 & 8,1 \\
\hline Comercio y reparaciones & 714 & 48,8 & 748 & 51,2 \\
\hline Hostelería & 301 & 44,3 & 379 & 55,7 \\
\hline Trasporte y almacenamiento & 358 & 80,3 & 88 & 19,7 \\
\hline Comunicación, actividades financieras, científicas y & 580 & 52,4 & 526 & 47,6 \\
administrativas & & & & \\
\hline Administración pública y educación y servicios sociales & 543 & 22,0 & 729 & 78,0 \\
\hline Actividades culturales deportivas y servicios personales & 296 & 31,5 & 643 & 68,5 \\
\hline
\end{tabular}

El análisis del nivel de estudios muestra una diferencia significativa $(p=0,000)$ en su distribución (tabla 6), en la que observamos que en los tramos de estudios superiores y licenciados universitarios es más elevado el porcentaje de mujeres. 
Tabla 6. Distribución de la muestra según nivel de estudios

\begin{tabular}{lrrrr}
\hline \multirow{2}{*}{ NIVEL DE ESTUDIOS } & \multicolumn{2}{c}{ HOMBRE } & \multicolumn{2}{c}{ MUJER } \\
\cline { 2 - 5 } & $\mathbf{n}$ & $\%$ & $\mathbf{n}$ & $\%$ \\
\hline No sabe leer ni escribir & 8 & 50,0 & 8,0 & 50,0 \\
Estudios primarios sin finalizar & 210 & 60,0 & 140 & 40,0 \\
\hline Estudios primarios (EGB, Graduado escolar, ESO, & 1.667 & 59,6 & 1.130 & 40,4 \\
Bachiller Superior, BUP, COU y equivalentes & & & & \\
\hline Formación Profesional primer grado. & 441 & 55,4 & 355 & 44.6 \\
\hline Formación Profesional segundo grado & 614 & 54,7 & 509 & 45,3 \\
\hline Bachillerato Superior, BUP, COU & 678 & 51,8 & 632 & 48,2 \\
\hline Estudios superiores de 2 o 3 años. Diplomado & 403 & 38,9 & 633 & 61,1 \\
\hline Licenciado Universitario. Arquitecto e Ingeniero Superior & 525 & 46,5 & 604 & 53,5 \\
\hline Doctorado y Estudios de postgrado o especialización & 147 & 51,9 & 136 & 48,1 \\
\hline Otros estudios no reglado & 16 & 72,7 & 6,0 & 27,3 \\
\hline
\end{tabular}

El análisis del horario de trabajo (tabla 7), evidencia que las mujeres predominaron en los siguientes tipos de horario: Fijo de mañana y turnos de mañana o tarde.

En el tipo de jornada, en la jornada a tiempo parcial tienen más peso las mujeres.

Esta diferente distribución entre géneros resultó estadísticamente significativa tanto para horario de trabajo, como para el tipo de jornada $(\mathrm{p}=0,0000)$.

Tabla 7. Distribución de la muestra según horario de trabajo, tipo de jornada y género

\begin{tabular}{lrrrr}
\hline \multirow{2}{*}{ HORARIO DE TRABAJO } & \multicolumn{2}{c}{ HOMBRE } & \multicolumn{2}{c}{ MUJER } \\
\cline { 2 - 5 } & $\mathbf{n}$ & $\%$ & $\mathbf{n}$ & $\%$ \\
\hline Jornada partida: mañana y tarde & 2.215 & 62,0 & 1.358 & 38,0 \\
\hline Fijo mañana & 1.047 & 41,1 & 1.501 & 58,9 \\
\hline Fijo tarde & 111 & 27,4 & 294 & 38,0 \\
\hline Fijo noche & 106 & 68,8 & 48 & 31,2 \\
\hline Turnos: mañana/ tarde & 688 & 53,2 & 606 & 46,8 \\
\hline Turnos: Mañana/Tarde /Noche & 399 & 62,0 & 245 & 38,0 \\
\hline Otro tipo de turno & 30 & 75,0 & 10 & 25,0 \\
\hline
\end{tabular}

En relación con el tipo de jornada, en la jornada a tiempo parcial tienen un mayor peso las mujeres.

Tabla 8. Distribución de la muestra según tipo de jornada y género

\begin{tabular}{lrrrr}
\hline & \multicolumn{2}{c}{ HOMBRE } & \multicolumn{2}{c}{ MUJER } \\
\cline { 2 - 5 } \multicolumn{1}{c}{ TIPO DE JORNADA } & n & $\%$ & n & $\%$ \\
\hline Jornada completa & 3.440 & 56,70 & 2.626 & 43,30 \\
\hline $\begin{array}{l}\text { Jornada parcial, no quiere ahora un trabajo de jornada } \\
\text { completa }\end{array}$ & 82 & 17,80 & 379 & 82,20 \\
\hline $\begin{array}{l}\text { Jornada parcial, no ha encontrado un trabajo de jornada } \\
\text { completa }\end{array}$ & 119 & 23,00 & 398 & 77,00 \\
\hline
\end{tabular}

Respecto al tipo de contrato (tabla 9), observamos una distribución desigual entre géneros $(\mathrm{p}<0,0005)$, predominando la mujer en los siguientes tipos de contratos: Interino, Fijo discontinuo y Temporal por ETT. 
Tabla 9. Distribución de la muestra según el tipo de contrato y género

\begin{tabular}{lrrrr}
\hline \multirow{2}{*}{ TIPO DE CONTRATO DE TRABAJO } & \multicolumn{2}{c}{ HOMBRE } & \multicolumn{2}{c}{ MUJER } \\
\cline { 2 - 5 } & \multicolumn{1}{c}{$\mathbf{n}$} & $\%$ & $\mathbf{n}$ & $\%$ \\
\hline Indefinido & 2.744 & 52,6 & 2.476 & 47,4 \\
\hline Fijo discontinuo & 192 & 46,5 & 221 & 53,5 \\
\hline Por obra o servicio & 481 & 52,5 & 435 & 47,5 \\
\hline Eventual por circunstancia de producción & 134 & 49,3 & 138 & 50,7 \\
\hline Interino & 49 & 32,5 & 102 & 67,5 \\
\hline De formación & 9 & 45,0 & 11 & 55,0 \\
\hline En prácticas & 9 & 56,3 & 7 & 43,8 \\
\hline Temporal por ETT & 18 & 48,6 & 19 & 51,4 \\
\hline
\end{tabular}

En relación con el análisis de la carga mental, se evaluaron los siguientes indicadores: "Nivel de Atención alto o muy alto», "Trabajar muy rápido», "Trabajar en plazos estrictos o cortos", "Tener mucho trabajo y sentirse agobiado".

Globalmente ambos géneros, tienen la percepción de que los factores de carga mental a los que están más frecuentemente sometidos son: "Nivel de Atención alto o muy alto" (Varones $=92,2 \%$, Mujer $=86,4 \%$ ) y "Trabajar muy rápido" (Varones= 61,9\% y Mujer $=65,9 \%)$.

El riesgo de estar sometido a los distintos factores de carga mental (tabla 10) varió entre géneros siendo estadísticamente superior en el hombre la exposición a: «Mantener un nivel de atención muy alto" y "Trabajar en plazos estrictos y cortos" $(\mathrm{OR}=1,87 \mathrm{y}$ $\mathrm{OR}=1,30$ respectivamente), siendo menor la magnitud, con respecto a la mujer, el hecho de "Trabajar muy rápido" $(\mathrm{OR}=0,89)$. Por el contrario "Tiene mucho trabajo y se siente agobiado" no existe diferencia significativa según género $(\mathrm{p}=0,088)$.

Tabla 10. Distribución según de los factores de carga mental: Nivel de atención, ritmo de trabajo, plazos y sentirse agobiado

\begin{tabular}{lcccccc}
\hline \multirow{2}{*}{ FACTORES DE CARGA MENTAL } & \multicolumn{2}{c}{ Hombre } & \multicolumn{2}{c}{ Mujer } & OR* & IC \\
\cline { 2 - 7 } & $\mathbf{n}$ & $\%$ & $\mathbf{n}$ & $\%$ & & \\
\hline Mantener un nivel de atención muy alto & 3.804 & 55,1 & 3.099 & 44,9 & 1,87 & $1,6-2,1$ \\
\hline Trabajar muy rápido & 2.119 & 51,8 & 1.974 & 48,2 & 0,89 & $0,8-0,9$ \\
\hline Trabajar con plazos muy estrictos y cortos & 1.738 & 56,1 & 1.362 & 43,9 & 1,3 & $1,2-1,4$ \\
\hline Tiene mucho trabajo y se siente agobiado & 1.105 & 52,0 & 1.021 & 48,0 & 0,9 & $0,8-1,0$ \\
\hline
\end{tabular}

Los datos de prevalencia de los distintos factores de carga mental, figuran en la tabla 11. Este análisis de prevalencia muestra un perfil de exposición muy similar entre géneros, reflejando unas cifras de prevalencias similares o superiores en el hombre.

Tabla 11. Prevalencia de los factores de carga mental, según género

\begin{tabular}{lcc}
\hline \multirow{2}{*}{ FACTORES DE CARGA MENTAL } & \multicolumn{2}{c}{ Prevalencia x 1000 } \\
\cline { 2 - 3 } & Hombre & Mujer \\
\hline Mantener un nivel de atención muy alto & 0,92 & 0,86 \\
Trabajar muy rápido & 0,63 & 0,65 \\
Trabajar con plazos muy estrictos y cortos & 0,49 & 0,42 \\
Tiene mucho trabajo y se siente agobiado & 0,35 & 0,37 \\
\hline
\end{tabular}


En relación al análisis de los factores protectores, se evaluaron los siguientes indicadores reflejados en la tabla 12»: "Ayuda de sus compañeros", "Ayuda de sus jefes", "Hacer lo que sabe hacer mejor», "Poner en prácticas sus propias ideas».

Destaca que el hombre alcanza en su trabajo mayor oportunidad de hacer aquello que sabe hacer mejor y realizar sus propias ideas $(\mathrm{OR}=1,4 \mathrm{y} O \mathrm{OR}=1,2)$. Por otra parte, el hecho de recibir apoyo o ayuda de superiores es elevado y muy semejante entre hombres y mujeres, encontrándose diferencias estadísticamente significativas, teniendo el hombre una menor oportunidad de ayuda de sus superiores $(\mathrm{OR}=0,9)$.

Tabla 12. Distribución de la muestra según factores protectores

\begin{tabular}{lcccccc}
\hline & \multicolumn{2}{c}{ Varón } & \multicolumn{2}{c}{ Mujer } & OR & IC \\
\cline { 2 - 7 } & $\mathbf{n}$ & $\%$ & $\mathbf{n}$ & $\%$ & & \\
\hline Ayuda de sus compañeros & 3.406 & 53,4 & 2.967 & 46,6 & 1,3 & $1-1,5$ \\
\hline Ayuda de sus superiores & 2.678 & 51,1 & 2.564 & 48,9 & 0,9 & $0,8-1$ \\
\hline $\begin{array}{l}\text { Tener la oportunidad de hacer aquello } \\
\text { que sabe hacer mejor }\end{array}$ & 3.415 & 54,6 & 2.841 & 45,4 & 1,4 & $1,2-1,6$ \\
\hline Poner en prácticas sus propias ideas & 2.934 & 54,8 & 2.424 & 45,2 & 1,2 & $1,1-1,3$ \\
\hline
\end{tabular}

En relación con la conciliación de la vida familiar y laboral (tabla 13), los hombres presentan de forma estadísticamente significativa $(\mathrm{p}=0,000)$, menos oportunidades de conciliación que la mujer.

Tabla 13. Distribución del grado de conciliación de la vida familiar y laboral por género

\begin{tabular}{lrcrcc}
\hline \multirow{2}{*}{ CONCILIACIÓN VIDA FAMILIAR Y LABORAL } & \multicolumn{3}{c}{ Varón } & \multicolumn{2}{c}{ Mujer } \\
\cline { 2 - 5 } & $\mathrm{n}$ & $\%$ & $\mathbf{n}$ & $\%$ \\
\hline Muy bien & 936 & 47,4 & 1.037 & 52,6 \\
\hline Bien & 2.651 & 54,2 & 2.239 & 45,8 \\
\hline No muy bien & 855 & 55,3 & 690 & 44,7 \\
\hline Nada bien & 268 & 57,9 & 195 & 42,1 \\
\hline
\end{tabular}

El resultado del análisis de regresión logística binaria puso de manifiesto que:

En el análisis de la carga mental por tener que mantener un nivel de atención elevado (tabla 14), la prueba de Hosmer- Lemeshow resultó no significativa $(\mathrm{p}=0,657)$, no difiriendo por tanto, los valores esperados por el modelo, con respecto a los observados.

Las variables que explican la existencia de un riesgo para este componente de carga mental fueron: trabajadores con estudios medios o superiores y trabajadores en el sector de la construcción.

Tabla 14. Análisis de carga mental debido al nivel de atención elevado

\begin{tabular}{lccc}
\hline \multicolumn{1}{c}{ CARGA DEBIDA AL NIVEL DE ATENCIÓN } & OR & \multicolumn{2}{c}{ 95\% C. I. } \\
\hline Género mujer & 0,625 & 0,468 & 0,833 \\
\hline Nivel estudios medios & 2,259 & 1,183 & 4,313 \\
\hline Nivel estudios superiores & 6,673 & 3,244 & 13,729 \\
\hline No existencia de delegado de prevención & 0,686 & 0,514 & 0,915 \\
\hline Jornada a tiempo parcial & 0,504 & 0,317 & 0,804 \\
\hline No disponer de apoyo de jefes o superiores & 0,691 & 0,497 & 0,961 \\
\hline Trabajar en sector agrario & 0,283 & 0,156 & 0,513 \\
\hline Trabajar en sector construcción & 4,653 & 1,632 & 13,270 \\
\hline
\end{tabular}


En el análisis de la carga mental por tener que trabajar muy rápido (tabla 15), La prueba de Hosmer- Lemeshow resultó no significativa $(\mathrm{p}=0,556)$, no difiriendo por tanto, los valores esperados por el modelo, con respecto a los observados.

Las variables que explican la existencia de un riesgo para este componente de carga mental fueron: ser mujer, trabajadores de empresas de más de 250 trabajadores, trabajadores extranjeros y trabajadores que no recibían apoyo de jefes.

Tabla 15. Análisis de carga mental debido a trabajar muy rápido

\begin{tabular}{lccc}
\hline \multicolumn{1}{c}{ TRABAJO MUY RÁPIDO } & OR & \multicolumn{2}{c}{ 95\% C. I. } \\
\hline Sexo mujer & 1,328 & 1,122 & 1,572 \\
\hline Empresas $>250$ trabajadores & 1,592 & 1,220 & 2,077 \\
\hline Trabajadores extranjeros & 1,629 & 1,142 & 2,325 \\
\hline No disponer de apoyo de jefes o superiores & 1,300 & 1,041 & 1,622 \\
\hline
\end{tabular}

En el análisis de la carga mental por tener que cumplir plazos muy estrictos (tabla 16), La prueba de Hosmer- Lemeshow resultó no significativa $(\mathrm{p}=0,747)$, no difiriendo por tanto, los valores esperados por el modelo con respecto a los observados.

Las variables que explican la existencia de un riesgo para este componente de carga mental fueron: trabajadores de empresas de más de 250 trabajadores, trabajadores con estudios superiores, trabajadores que no recibían apoyo de jefes y trabajadores de la construcción. Presentan un menor riesgo los trabajadores en jornada a tiempo parcial.

Tabla 16. Análisis de carga mental debido a trabajar en plazos muy estrictos

\begin{tabular}{llll}
\hline & OR & 95\% C. I. \\
\hline Empresas $>$ 250 trabajadores & 1,826 & 1,432 & 2,329 \\
\hline Nivel de estudios superiores & 1,617 & 1,009 & 2,591 \\
\hline Jornada a tiempo parcial & 0,672 & 0,487 & 0,928 \\
\hline No disponer de apoyo de jefes o superiores & 1,351 & 1,105 & 1,651 \\
\hline Trabajar en sector construcción & 2,306 & 1,674 & 3,176 \\
\hline
\end{tabular}

En el análisis de la carga mental por tener mucho trabajo (tabla 17), la prueba de Hosmer- Lemeshow resultó no significativa $(\mathrm{p}=0,482)$, no difiriendo por tanto, los valores esperados por el modelo, con respecto a los observados.

Las variables que explican la existencia de un riesgo para este componente de carga mental fueron: ser mujer, trabajar en empresas de más de 250 trabajadores, nivel de estudios superiores, no recibir apoyo de jefes

Presentan un menor riesgo los trabajadores con contrato temporal, trabajadores en jornada a tiempo parcial, trabajar en los sectores agrario e industria.

Tabla 17. Análisis de carga mental debido a tener mucho trabajo

\begin{tabular}{lccc}
\hline & OR & \multicolumn{2}{c}{ 95\% C. I. } \\
\hline Sexo mujer & 1,280 & 1,079 & 1,520 \\
\hline Empresas> 250 trabajadores & 1,636 & 1,247 & 2,146 \\
\hline Nivel de estudios superiores & 2,078 & 1,200 & 3,600 \\
\hline Contrato temporal & 0,679 & 0,539 & 0,855 \\
\hline Jornada a tiempo parcial & 0,555 & 0,381 & 0,810 \\
\hline No disponer de apoyo de jefes o superiores & 1,370 & 1,107 & 1,695 \\
\hline Trabajar en sector agrario & 0,546 & 0,302 & 0,987 \\
\hline Trabajar en sector industria & 0,738 & 0,591 & 0,920 \\
\hline
\end{tabular}




\section{DISCUSIÓN Y CONCLUSIONES}

Los resultados del análisis no nos permiten corroborar la hipótesis de la existencia de un mayor «Factor de carga mental» global en la mujer.

En relación con los objetivos planteados en el estudio, "Ser mujer", supuso un factor de riesgo para los componentes de la carga mental derivada de "Trabajar muy rápido" y "Tener mucho trabajo", presentando un nivel de carga menor que el hombre para la carga mental derivada de tener que "Mantener un nivel de atención muy alto".

El género no influyó en el componente de carga relativo a tener que «Trabajar con plazos muy estrictos y cortos».

Superaron la prevalencia en la mujer sobre el varón los factores de «Trabajar muy rápido" $\mathrm{y}$ "Tiene mucho trabajo y se siente agobiado".

Un dato que se repite en encuestas anteriores es el referente a los ritmos de trabajo. La mujer debe trabajar a un ritmo más rápido. Esto puede ser debido, a la relación directa con los empleos que desarrolla: en mayor medida los de menos responsabilidad y más contratos de tipo parcial y eventuales.

También afirman poder conciliar, mejor que el hombre, la vida familiar y laboral, hecho que puede ser debido al tipo de jornada (tiempo parcial) o tipo de relación laboral (temporalidad). Así en las mujeres es más frecuente el contrato interino $67,5 \%$, lo cual también puede ser una causa que ahonde en esta situación. En cuanto a las diferencias entre hombres y mujeres, es notable el hecho de que la temporalidad femenina es claramente superior a la masculina, si bien las dos tasas han tendido a acercarse en los últimos años ${ }^{12}$.

Estos factores (temporalidad y reducciones de jornadas) se hacen más evidentes en periodos de crisis económicas y es mayor entre las mujeres, que aunque suele ser más acusado entre las peor situadas, también afecta a las más cualificadas ${ }^{18}$.

Respecto a los horarios de trabajo, comparando con los resultados de la VI Encuesta de Condiciones de Trabajo $^{10}$ se repite el dato, que la mujer tiene con más frecuencia jornada continuada fijo/mañana o fijo/tarde. Posiblemente está relacionado con el tiempo de ocupación diario que la mujer debe emplear en la vida familiar, siendo este tipo de turno el que compagina mejor con sus necesidades ${ }^{21}$.

Los resultados ponen en evidencia la mayor proporción de mujeres que han realizado estudios superiores con respecto al hombre, lo que contrasta con el hecho de que a medida que avanzamos en la escala profesional, se observa una disminución de mujeres en cargos de mayor responsabilidad. Por citar sólo un dato de situación en este aspecto, sobre el total del personal empleado en las instituciones públicas, un $20 \%$ del total de hombres están dentro del grupo de Grado A (catedráticos y otros cargos de mayor responsabilidad), mientras que entre las mujeres ese porcentaje es tan sólo del 3\% ${ }^{18}$.

A pesar de una mayor formación, detectamos que la mujer tiene menos posibilidades de hacer aquello que sabe hacer mejor y de llevar a cabo sus propias ideas. Quizás este aspecto sea un motivo más por el que la mujer abandone la vida laboral e incluso que prefiera no asumir cargos de mayor responsabilidad, porque ello implicaría menor dedicación a la familia.

Observamos que a partir de los 30- 40 años comienza a descender la presencia de la mujer en la vida laboral ${ }^{18}$.

Puede pensarse en varias causas (abandono de la vida laboral), como es el hecho de que las mujeres tienen menos oportunidades de trabajo, más probabilidad de ser excluidas del mercado laboral así como de las estrategias de protección social ${ }^{19}$.

Otro dato que podría explicar este hecho, sería que esa época de la vida coincide con el cuidado de los hijos y en muchos de casos es frecuente la primera maternidad en 
ese rango de edad. Cuando las mujeres llegan a ese momento de su vida, ante la necesidad de atender la vida familiar y laboral se sienten culpables, algunas deciden reducir o suprimir el tiempo que dedican al trabajo productivo, lo que no sucede con los hombres o al menos en una menor proporción ${ }^{20}$.

Las mujeres que optan por abandonar el empleo o asumir jornadas parciales, manifiestan una clara orientación familiar cuando se encuentran ante la disyuntiva de combinar empleo y familia. ${ }^{21}$ Esta reflexión sintoniza con los resultados de nuestro trabajo, coincidiendo que la jornada parcial es mucho más frecuente en la mujer.

En el análisis multivariante, podemos observar que para los componentes de la carga mental derivada de "Trabajar muy rápido" se observa que está más presente en la mujer que en el hombre de forma significativa. Esto podría ser un factor añadido que justificara el abandono de la mujer del mercado del trabajo.

Las condiciones laborales no son las más idóneas para motivar la continuidad en el mundo laboral, ya que el puesto que ocupan es en muchas ocasiones de menor categoría al de su preparación y conocimientos, suponiendo una menor realización personal.

También se pone de manifiesto que la mujer siente que «Tiene mucho trabajo y se siente agobiada", podríamos pensar que sería unos de los factores que lleva a la mujer con mayor frecuencia a realizar un tipo de jornada partida, para compaginar de una manera más equilibrada los esfuerzos que realiza en la vida laboral y familiar.

Sin embargo, en el análisis de las variables asociadas con carga mental por tener que mantener un nivel de atención muy alto, el ser mujer no está relacionado con mayor carga mental, como sí le supone al hombre. Posiblemente esto refuerza el dato de que la mujer ocupa puestos de trabajo que requieren menor nivel de atención en su tarea.

Existen pocos estudios desde la perspectiva de género, que permitan comprobar si la situación laboral y condiciones de carga mental y factores psicosociales que existen en la actualidad en el ámbito laboral, se adaptan a la situación particular de la mujer. Por lo que creemos que este estudio tiene mucha relevancia de cara a tenerlo en cuenta para mejorar las condiciones laborales de la mujer.

La crisis actual añade además que tal reforzamiento de las desigualdades afecta al futuro de las sociedades del bienestar, que era donde las mujeres habían alcanzado mayores logros. Siendo así que la crisis está haciendo evidente la fragilidad de tales logros, aunque sea cierto que no solo son esos logros los que están en riesgo. Una desregulación que se concreta en un tipo de jornada que queda lejos de la reducción del tiempo de trabajo. En la actualidad, se convierte en la senda que lleva a las mujeres a tener peores salarios, nula posibilidad de desarrollar una carrera profesional, si es que pueden permitírselo, y peores pensiones en un futuro cada vez más incierto. Al tiempo que les impide compatibilizar su actividad laboral con el trabajo doméstico y de cuidados, que deben asumir como si de algo innato se tratara ${ }^{18}$.

Los datos muestran la continuidad de la segregación ocupacional. Segregación horizontal, visualizada a través de la concentración del empleo femenino en los sectores de actividad con menor prestigio y peores condiciones laborales. Segregación vertical, expresada a través de la desigual, presencia de mujeres y hombres en la jerarquía empresarial, con casi nula presencia femenina en las cúpulas directivas. Lo que viene a ser lo mismo, que las mujeres están muy concentradas en pocas ocupaciones, la mayoría de ellas relacionadas con el cuidado de la vida, la limpieza, y tareas administrativas. Mientras los índices de masculinidad se reparten entre un mayor y más variado número de ocupaciones ${ }^{18}$.

Parece viable reconocer que las desigualdades de género en el mercado de trabajo no desaparecen sino que se transforman. En este sentido, los datos aportados defienden la perpetuación de las desigualdades de género que se transforman al ritmo que se desregula y precariza el mercado laboral. Unas desigualdades que se tornan más evidentes entre los colectivos de mujeres peor situadas, pero que no desaparecen entre aquellas 
mujeres más cualificadas y en mejor posición de partida para lograr un empleo en igualdad de condiciones al de sus compañeros masculinos ${ }^{18}$.

Las fortalezas de los resultados de este estudio se fundamentan en la fiabilidad de los datos de la VII Encuesta Nacional de Condiciones de Trabajo ${ }^{17}$, lo que permite estudiar la desigual de género desde una base poblacional.

La principal limitación es el tipo de diseño, ya que al ser un estudio transversal en el que valoramos prevalencia no se pueden establecer relaciones causales.

Puede existir un sesgo de autoselección dado que se trata de una encuesta de participación voluntaria, que puede estar minimizado por el gran tamaño muestral.

\section{BIBLIOGRAFÍA}

1. Cuarta Conferencia Mundial sobre la Mujer [acceso 20 de junio de 2014]. Disponible en: http://www. un.org/womenwatch/daw/beijing/pdf/Beijing\%20full\%20report\%20S.pdf

2. Tratado de Amsterdan [acceso 20 de junio de 2014]. Disponible en: http://www.europarl.europa.eu/ topics/treaty/pdf/amst-es.

3. Guía para la incorporación de la perspectiva de género [acceso 7 de junio de 2012]. Instituto de la Mujer. España. Disponible en: SGPROGmujer@mtas.es

4. Manual para la perspectiva de género en las políticas de empleo de inclusión social y de protección social. [acceso 9 de junio de 2012] Dirección General de Empleo, Asuntos Sociales e Igualdad de Oportunidades de la Comisión Europea.2008 Disponible en: http://ec.europa.eu/social/BlobServlet?docId=2045\&langId=es

5. Ley Orgánica 3/2007, de 22 de marzo, para la igualdad efectiva de mujeres y hombres. BOE n. 71 .

6. Gender Equality and Decent Work. 2012 [acceso 20 de junio de 2014]. Disponible en: http://www.ilo.org/ wcmsp5/groups/public/---ed_norm/-normes/documents/publication/wcms

7. La mujer y la salud en el trabajo. [20 de junio de 2012] Agencia Europea para la Seguridad y la Salud en el Trabajo. Disponible en: (http://osha.europa.eu/es/priority_groups/gender).

8. Forastieri. V. Women workers and gender issues on occupational safety and health-information note. [ 31 de julio de 2012]. Disponible en: (http://www.ilo.org/safework/info/publications/WCMS_108003/lang--n/ index.htm

9. Wu SY, Li HY, Wang XR, Yang SJ, Qiu H. Arch. A comparison of the effect of work stress on burnout and quality of life between female nurses and female doctors Environ Occup Health.2011; 66 (4):193-200.

10. VI Encuesta nacional de condiciones de Trabajo [acceso 7 de junio de 2012] INSHT. Disponible http:// www.insht.es/).

11. 1. ${ }^{a}$ Conferencia mundial sobre la mujer. Confederación Sindical Internacional [acceso 20de junio de 2012] Bruselas 2009. Disponible en: / http://www.ituc-csi.org/1a-cmpm-conclusiones

12. Toharia L El mercado de trabajo en España: situación y perspectivas. C L M. Economía, 2004(4): 77-106 ? MIRAR $1 .^{\circ}$ SEMESTRE

13. Samuelsson $\AA$, Houkes I, P Verdonk, Hammarström A. Types of employment and their associations with work characteristics and health in Swedish women and men Suecia. Scand J Public Health.2012;40(2):183-90

14. García Calvente, M. Guía para incorporar la perspectiva de género a la investigación en salud [acceso 20 de junio de 2012]. Escuela Andaluza de Salud Pública. (http://www.easp.es).

15. Mota NP, Medved M, Wanq J, Asmundson GJ, Whitney D, J Sareen. Stress and mental disorders in female military personnel: comparisons between the sexes in a male dominated profession. J Psychiatr Res. 2012; 46(2):159-67.

16. Campos-Serna, J., Ronda-Pérez, E., Artazcoz, L., Benavides. Desigualdades de género en salud laboral en España. Gac. Sanit.2012 (605): 1-9

17. VII Encuesta Nacional de Condiciones de Trabajo [acceso 9 de septiembre de 2012]. Instituto Nacional de Seguridad e Higiene del Trabajo (http://www.insht.es/).

18. Torns, T., Recio, C. Las desigualdades de género en el mercado de trabajo entre la continuidad y la transformación [4 de agosto de 2014] Rev. Económic Critica 2012;14:178-202. Disponible en: http:// revistaeconomiacritica.org/sites/default/files/revistas/n14/Semimonografico 
19. Sabates -Wheeler,. Kabeer. N.2009 Genter equality and the extensin of social protection [acceso 20 de junio de 2014] ILO Social Protection Department (SOCPR). Disponible en: http://www.ilo.org/secsoc/ information-resources/publications-and-tools/Workingpapers/WCMS_207686/lang--en/index.htm

20. Rodríguez Menéndez, M.C., Fernández García, C. Cuadernos de Relaciones Laborales Empleo y maternidad: el discurso femenino sobre las dificultades para conciliar familia y trabajo, vol. 28, núm. 2 (2010) 257-275

21. VI Encuesta Nacional de Condiciones de Trabajo Ministerio de Trabajo y Asuntos Sociales. Disponible en: http://prevencion.wordpress.com/2007/11/23/vi-encuesta-nacional-de-condiciones-de-trabajo/encuesta

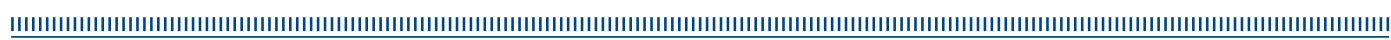

